\title{
Design of Educational Instantaneous Powers Metering Software for Steady-State Single-Phase AC Networks
}

\author{
Rene Wamkeue, Senior Member, IEEE, \\ UQAT, Québec \\ rene.wamkeue@uqat.ca
}

\author{
Theubou Tameghe Tommy Andy, Nyobe Yome Jean Maurice, \\ UQAT, Québec \\ ENSET, Douala, CMR \\ Tommy-andy.theubou-tameghe@uqat.ca nyobeyome@yahoo.fr
}

\begin{abstract}
In this paper, instantaneous complex current and voltage for steady-state single-phase alternating-current (AC) circuit are introduced to improve the teaching methodology of instantaneous powers in undergraduate power courses. In contrast to the classical approach using fixed phasors, it is shown in this paper that an instantaneous solution approach also provides interesting results and leads to exact formulations of instantaneous powers. As a step towards building instantaneous reactive-power metering equipment for teaching purposes, a numerical model has been developed using Matlab/Simulink software. The evaluation of the approach by means of a student assessment is also addressed in the paper.
\end{abstract}

Keywords: Steady-state AC circuits, rotating phasors, instantaneous powers, instantaneous powers metering software, students assessments and simulations

\section{Introduction}

For many years, the phasor representation described in many electric circuit text books [1]-[4], and generally credited to Charles Proteus Steinmetz, an electrical engineer for General Electric, has been particularly helpful for visualizing and teaching steady-state phenomena in analyzing networks with sinusoidal signals [5]. However, the method as currently used does not provide clear answers to frequently asked students' questions such as: (1) Does the definition of power given by $p$ $(\mathrm{t})=\mathrm{e}(\mathrm{t}) \mathrm{i}(\mathrm{t})$ for a given network include the instantaneous reactive power? (2) Is it the instantaneous real power or instantaneous apparent power? (3) Does the reactive instantaneous power for single-phase AC circuits exist? If the answer is yes, what is its comprehensible definition? The most recent answers to these fundamental questions can be found in [5]-[6] and have been expanded by some colleagues in their text books [7]. Many works have been proposed for instantaneous powers formulations in non-sinusoidal networks for power compensation [8]-[13]. No attempt was made at providing clear definitions of instantaneous powers for timedomain steady-state single phase AC networks. This leads to much confusion. Furthermore, as will be demonstrated in this paper, the classical theory presents some mathematical shortcomings.

The instantaneous complex apparent power is used in this paper to derive the instantaneous active and reactive power. An attempt is made to propose a more comprehensible physical interpretation for each power component (apparent, active and reactive powers) and thus to provide clear answers to common questions that have not yet been answered. The main objectives of the paper are:
- To summarize the solution procedure for a steady-state alternating-current (AC) network using fixed phasors

- To proposed new concepts of instantaneous active, reactive and apparent powers using the rotating or instantaneous current phasors

- To provide the student with a graphical interpretation of the instantaneous reactive power together with related curves for various types of load

- To prove the effectiveness of the proposed theory by means of comparisons of numerical simulation results as classroom activities using a Matlab/Simulink software model

- To further assess the effectiveness of the teaching approach with a student feedback questionnaire obtained in an Electrotechnology course with 15 participants.

\section{Phasors in steady-state AC circuits}

If the sinusoidal voltage (1) supplies an AC load (an example of an R,L load is given in Figure 1), this will produce a sinusoidal current given by (2) as the solution of the Kirchhoff mesh differential equation (3) of the circuit illustrated in Figure 1 [1]-[3].

$$
\begin{aligned}
& e(t)=E_{m} \cos \left(\omega t+\theta_{e}\right) \\
& i(t)=I_{m} \cos \left(\omega t+\theta_{i}\right) \\
& e(t)=\operatorname{Ri}(t)+L \frac{d i(t)}{d t} \\
& e(t)=\operatorname{Re} \bar{e}(t)=\operatorname{Re} E_{m} e^{j \omega t+\theta_{e}} \\
& i(t)=\operatorname{Re} \bar{i}(t)=\operatorname{Re}\left(I_{m} e^{j\left(\omega t+\theta_{i}\right)}\right)
\end{aligned}
$$

The voltage and current in (1) and (2) can be seen as real components of fictitious rotating phasors (4).

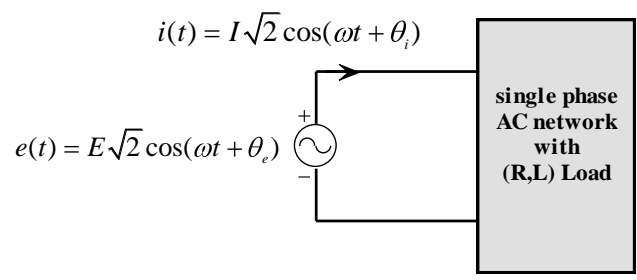

Figure 1. Single-phase AC network with RL load

\section{Solution of AC circuit using fixed phasors}

Solving the differential equation (3) for a circuit with more than one mesh can be tedious. In order to avoid this, the differential equation (3) can be transformed into a related algebraic equation (7) by simultaneously solving the real 
differential and related imaginary equations as organized in (5), which is equivalent to (6).

$$
\begin{gathered}
\left\{\begin{array}{l}
\operatorname{Re} \bar{e}(t)=R \operatorname{Re} \bar{i}(t)+L \frac{d}{d t} \operatorname{Re} \bar{i}(t) \\
\operatorname{Im} \bar{e}(t)=R \operatorname{Im} \bar{i}(t)+L \frac{d}{d t} \operatorname{Im} \bar{i}(t)
\end{array}\right. \\
\operatorname{Re} \bar{e}(t)+j \operatorname{Im} \bar{e}(t)=R \operatorname{Re} \bar{i}(t)+j \operatorname{Im} \bar{i}(t) \\
+L \frac{d}{d t} \operatorname{Re} \bar{i}(t)+j \operatorname{Im} \bar{i}(t) \\
=\operatorname{Re}\left[R \bar{i}(t)+L \frac{d}{d t} \bar{i}(t)\right]+j \operatorname{Im}\left[R \bar{i}(t)+L \frac{d}{d t} \bar{i}(t)\right] \\
\bar{e}(t)=R \bar{i}(t)+L \frac{d}{d t} \bar{i}(t) \\
E e^{j \theta_{e}} \sqrt{2} e^{j \omega t}=\left(R I e^{j \theta_{i}}+j L \omega I e^{j \theta_{i}}\right) \sqrt{2} e^{j \omega t} \\
E e^{j \theta_{e}}=R+j L \omega I e^{j \theta_{i}} \Leftrightarrow \bar{E}=R+j L \omega \bar{I}
\end{gathered}
$$

Solving (3) is mathematically equivalent to solving the algebraic complex equation (7), which can be transformed as defined in (8) and (9). Equation (9) is associated with the classical approach using fixed constant phasors [3], [4], while (8) is related to the instantaneous phasors proposed in this paper. Complex numbers $\bar{E}=E \angle \theta_{e}$ and $\bar{I}=I \angle \theta_{i}$ are called constant phasors (in contrast to the so-called rotating rotating phasors) or simply phasors (in common usage) of the instantaneous voltage (1) and current (2) respectively. In timedomain steady-state analysis of AC circuit, the source voltage is a control input variable (the source is supplied by the users) which can easily be handled. For a given real source voltage $e(t)=E_{m} \cos \left(\omega t+\theta_{e}\right)$, the voltage angle $\theta_{e}$ can be fixed as zero angle reference $\left(\theta_{e}=0^{0}\right)$. This greatly simplifies the network analysis. In such a context, $\bar{E}=E \angle 0^{0}$ and $e(t)=E_{m} \cos (\omega t)$ for fixed and instantaneous phasors analysis respectively; thus, the solution of (9) yield:

$$
\begin{aligned}
& \bar{E}=E \angle 0^{0} \\
& \bar{I}=I \angle-\varphi=I \cos \varphi-j I \sin \varphi=I_{\text {active }}-j I_{\text {reactive }} \\
& \varphi=\tan ^{-1}(L \omega / R)=\theta_{e}-\theta_{i}=-\theta_{i} \geq 0 \\
& I=\sqrt{I_{\text {active }}^{2}+I_{\text {reactive }}^{2}}
\end{aligned}
$$

A single phase AC circuit with an $\mathrm{R}, \mathrm{L}$ load supplied by a sinusoidal voltage of phasor given by (10) is crossing by an active (real) and reactive (imaginary) current defined by first equation of (10). The complexe representation of the network current is illustrated in Fig.2 where the supplied voltage is on the real axis.

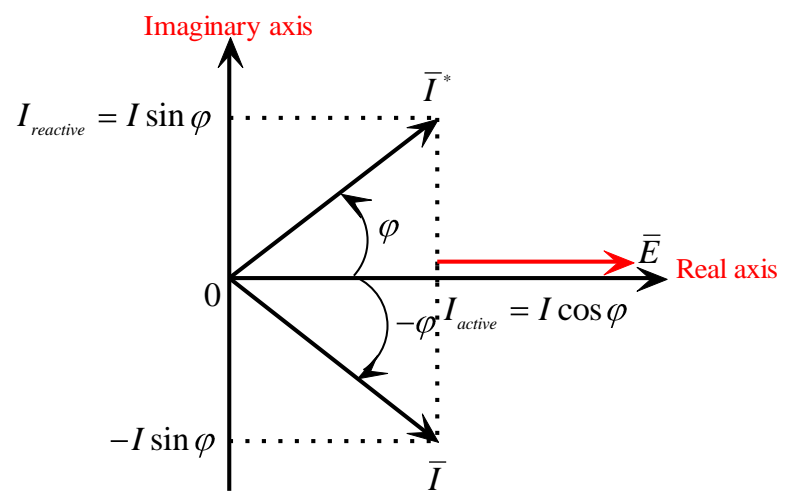

Figure 2. Network current fixed phasor diagram with illustration of its active and reactive components

Active and reactive powers are defined using voltage and current fixed phasors as real and imaginary parts of the complex apparent power (12) respectively. The power diagram in Figure 3 is obtained by multiplying the current diagram (Figure 2) by the reference real voltage $\bar{E}=E$.

$$
\begin{aligned}
& \bar{S}=E \bar{I}^{*}=P+j Q=E I \cos \varphi+j I \sin \varphi \\
& =E I_{\text {active }}+j E I_{\text {reactive }}=\operatorname{Re}(\bar{S})+j \operatorname{Im}(\bar{S})
\end{aligned}
$$

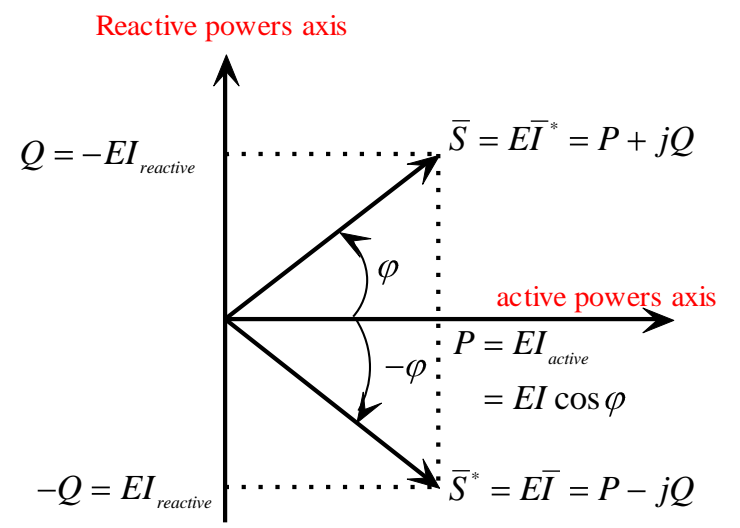

Figure 3. Fixed complex apparent, active and reactive power diagram

Expression (12) can be interpreted by saying that the active and reactive powers are due respectively to the real and imaginary parts of the network current.

\section{Solution of AC circuit using rotating phasors: New approach}

In the previous analysis with fixed phasors, the supplied voltage $\bar{E}=E \angle 0^{0} \quad$ (10) is a real number and the related current (11) is a complex number. Similarly, the instantaneous solution can be developed from (8) using rotating phasors. In fact, (8) can be reformulated as defined in (13) with the rotating-current solution given by (14). 


$$
\begin{aligned}
& E_{m} e^{j\left(\omega t+\theta_{e}\right)}=R+j L \omega\left(I_{m} e^{j\left(\omega t+\theta_{i}\right)}\right) \\
& \bar{e}(t)=R+j L \omega \bar{i}(t) \\
& \bar{i}(t)=I_{m} \angle \omega t-\varphi=I_{m} \cos \omega t-\varphi+j I_{m} \sin \omega t-\varphi \\
& =i_{\text {active }}(t)+j i_{\text {reactive }}(t) \\
& \varphi=\tan ^{-1}(L \omega / R)=\theta_{e}-\theta_{i}=0-\theta_{i} \geq 0 \\
& I_{m}=\sqrt{i_{\text {active }}^{2}+i_{\text {reactive }}^{2}} \\
& \bar{e}(t)=e(t)=E_{m} \cos \omega t \\
& \bar{s}(t)=e(t) \bar{i}^{*}(t)=p(t)+j q(t) \\
& =E_{m} \cos \omega t\left[I_{m} \cos \omega t-\varphi-j I_{m} \sin \omega t-\varphi\right] \\
& p(t)=\operatorname{Re}[\bar{s}(t)]=e(t) i(t)=e(t) i_{\text {active }}(t) \\
& =E_{m} I_{m} \cos \omega t \cos (\omega t-\varphi) \\
& q(t)=\operatorname{Im}[\bar{s}(t)]=-e(t) i_{\text {reactive }}(t) \\
& =-e(t) i\left(t-\frac{\pi}{2 \omega}\right)=-E_{m} I_{m} \cos \omega t \sin (\omega t-\varphi) \\
& \bar{i}_{\text {reactive }}=-I \sqrt{2} \sin (\omega t-\varphi)
\end{aligned}
$$

Figure 4. Network rotating current phasor diagram with illustration of its active and reactive components

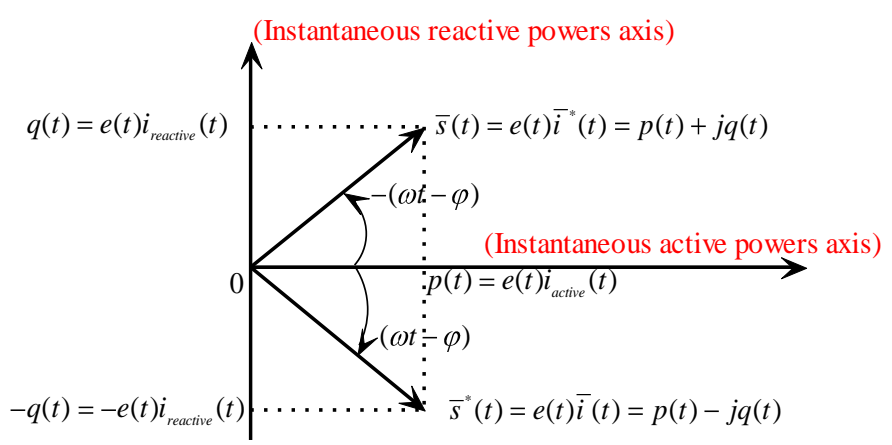

Figure 5. Instantaneous rotating complex apparent, active and reactive power diagram

Figure 4 illustrates different components of the instantaneous complex current $\bar{i}(t)(14)$ (also called the rotating current phasor). As shown in section 3 , in the rotating phasor reference frame, the supplied reference voltage is given by (15). The input reference voltage should necessarily be a real number as previously stated in the fixed-phasor analysis since it's a control variable. In fact, the supplied voltage (15) pulsates only on the real axis at $\omega \mathrm{rad} / \mathrm{s}$. As previously expressed in (12) for fixed phasors, the instantaneous complex apparent power is defined by (16) and the graphical explanation is given in Figure 5. The instantaneous active (17) and reactive (18) powers are defined as real and imaginary parts of the apparent power (16) respectively. The instantaneous active and reactive powers obtained in (17) and (18) can be developed as given in (19) and (20) respectively. It is interesting to observe that in the new approach instantaneous active and reactive powers defined by (19) and (20) play similar roles. In fact, (19) and (20) can be physically interpreted by saying that in a steady-state singlephase AC network with combined active/reactive equivalent load, the active part of the load consumes a DC active power called the 'average active power'; similarly, the reactive part of the load consumes $(\varphi>0)$ or delivers $(\varphi<0)$ a DC reactive power called the 'average reactive power'. Zero mean active $p_{2 \omega}(t)$ and reactive $q_{2 \omega}(t)$ powers defined respectively in (19) and (20) fluctuate in the network load independently from the nature of this load, which only creates a delay angle $\varphi$ with the supplied voltage.

$$
\begin{aligned}
p(t) & =\overbrace{E I \cos \varphi}^{\text {average active power }}+\overbrace{E I \cos 2 \omega t-\varphi}^{\begin{array}{c}
\text { zero mean fluctuated } \\
\text { active power }
\end{array}} \\
& =P+\overbrace{p_{2 \omega}(t)}^{\text {average reactive power }} \\
q(t) & =\overbrace{E I \sin \varphi}^{\begin{array}{c}
\text { cero mean fluctuated } \\
\text { reactive power }
\end{array}} \\
& =Q+\overbrace{E I \text { sin } 2 \omega t-\varphi}^{2 \omega t}
\end{aligned}
$$

The following well known quantities can be derived from (19) to (21) of the proposed approach:

$$
\begin{aligned}
& P=\frac{1}{2 \pi} \int_{0}^{2 \pi} p(\omega t) d(\omega t)=E I \cos \varphi \\
& Q=\frac{1}{2 \pi} \int_{0}^{2 \pi} q(\omega t) d(\omega t)=E I \sin \varphi \\
& \bar{S}=\frac{1}{2 \pi} \int_{0}^{2 \pi} \bar{S}(\omega t) d(\omega t)=P+j Q \\
& S=E I=\sqrt{P^{2}+Q^{2}}
\end{aligned}
$$

The new definition of the reactive (23) and complex apparent (24) powers seems mathematically more coherent and physically consistent than the common currently accepted theory.

\section{Utilization of the new theory with elementary $R, L$ and $C$ loads}

In this section, the new definitions of power in a steady-state single phase AC network are studied for each single elementary load (resistive, inductive and capacitive). 


\subsection{Case of purely resistive load $\left(\varphi=0^{0}\right)$}

For a purely resistive load, (22)-(24) become:

$$
\begin{gathered}
p(t)=E I+E I \cos 2 \omega t=P+p_{2 \omega}(t) \\
q(t)=0-E I \sin 2 \omega t=0+q_{2 \omega}(t) \\
\bar{S}(t)=\bar{S}+\bar{S}_{2 \omega}(t)=P+j 0+p_{2 \omega}(t)+j q_{2 \omega}(t)
\end{gathered}
$$

The zero average reactive power given in (27) for a purely resistive load is close to the commonly accepted theory, which indicates a zero fluctuated reactive power for a purely resistive load. But, in this case $q_{2 \omega}(t)$ exists and is a non-zero function even if its mean is null. This result reveals that a purely resistive load consumes an active average power and is also subjected to active and reactive fluctuated powers of frequency $2 \omega$. Figure 6 illustrates the active and reactive instantaneous power waveforms for a resistive load. The average reactive power $\mathrm{Q}$ is zero (Figure 6).

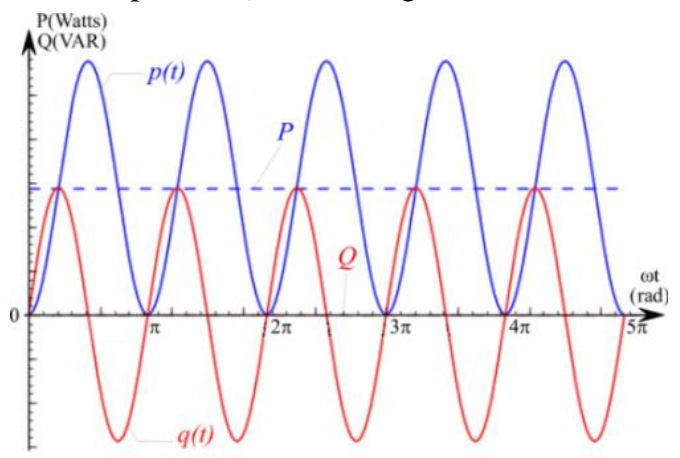

Figure 6. Instantaneous active and reactive powers following a purely resistive load

5.2 Case of purely inductive $\left(\varphi=90^{0}\right)$ or capacitive $\left(\varphi=-90^{0}\right)$ Load

For a purely inductive or capacitive load, (22)-(24) become:

$$
\begin{gathered}
p(t)=0 \pm E I \sin 2 \omega t=P+p_{2 \omega}(t) \\
q(t)= \pm E I \mp E I \cos 2 \omega t=Q+q_{2 \omega}(t) \\
\bar{s}(t)=0+j Q+p_{2_{\omega}}(t)+j q_{2_{\omega}}(t)
\end{gathered}
$$

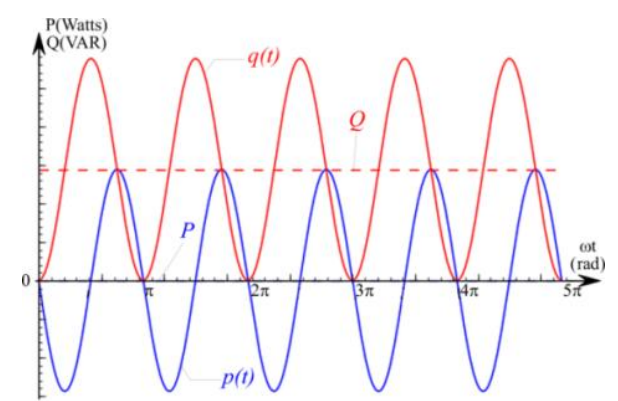

Figure 7. Instantaneous active and reactive powers following a purely inductive load
As above for a resistive load, the zero average active power given in (29) for a purely inductive or capacitive load, is close to the common theory. In contrast to the theory developed in [6]-[8], $p_{2 \omega}(t)$ exists and is a non-zero function even if its mean is null. This also points out that a purely inductive or capacitive load respectively consumes or delivers a reactive average power and is also subjected to active and reactive fluctuated powers of frequency $2 \omega$ as given by (29) and (30), (Figures 7 and 8).

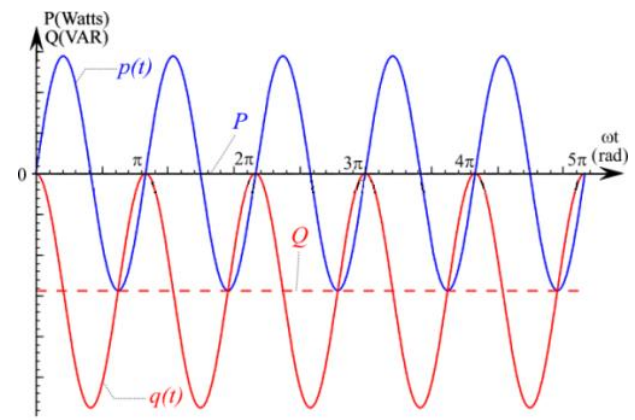

Figure 8. Instantaneous active and reactive powers following a purely capacitive load

\section{Instantaneous powers metering software}

As an important step towards building instantaneous reactivepower metering equipment for teaching purposes, a numerical model illustrated by the block diagram in Fig. 16 was developed using Matlab/Simulink software. For model assessment, direct computation of analytical power formulas using Matlab programs was done for comparison with the Matlab/Simulink numerical model, as shown in Table1 where different power factor RLC series loads were chosen.

\begin{tabular}{|c|c|c|c|c|c|}
\hline Conditions & \multicolumn{5}{|c|}{$\mathrm{V}=120 \mathrm{Vac} / 60 \mathrm{~Hz} ; \mathrm{I}=9.2 \mathrm{~A} ; \mathrm{k}=3.64 \times 10^{3}$} \\
\hline \multirow{3}{*}{$\begin{array}{l}\text { (Matlab) } \\
\text { Results from } \\
\text { analytical } \\
\text { formulas }\end{array}$} & $\varphi\left(^{\circ}\right)$ & 85.6 & -85.6 & 0.0 & 45.0 \\
\hline & $\mathrm{P}(\mathrm{W})$ & 84.88 & 84.65 & 1104 & 780.6 \\
\hline & $\begin{array}{c}\mathrm{Q} \\
\text { (VAR) }\end{array}$ & 1101 & -1101 & 0 & 780.6 \\
\hline \multirow{2}{*}{$\begin{array}{l}\text { Matlab/Simulink } \\
\text { model results }\end{array}$} & $\begin{array}{l}\mathrm{P}_{\text {mean }} \\
(\mathrm{W})\end{array}$ & 84.82 & 84.82 & 1104.0 & 780.8 \\
\hline & $\begin{array}{l}\mathrm{Q}_{\text {mean }} \\
(\mathrm{W})\end{array}$ & 1100 & -1238.1 & -10.55 & 780.8 \\
\hline
\end{tabular}

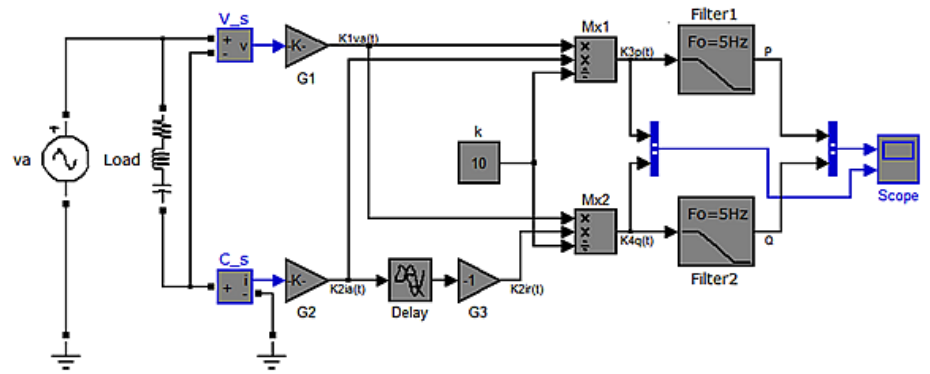

Figure 9. Matlab/Simulink model of an instantaneous power metering system

Table 1. Average active and reactive powers for various load conditions

Table 1 and Figure 10 clearly confirm the accuracy of the Matlab/Simulink numerical model. The small discrepancy observed between the analytical formulations and the 
Matlab/Simulink numerical results can be explained as internal solver approximations of the Matlab/Simulink software. A RLC load with a load angle $\varphi=85.6^{\circ}$ and the initial conditions given in Table 1 were used for the simulation results provided in Figure 10.

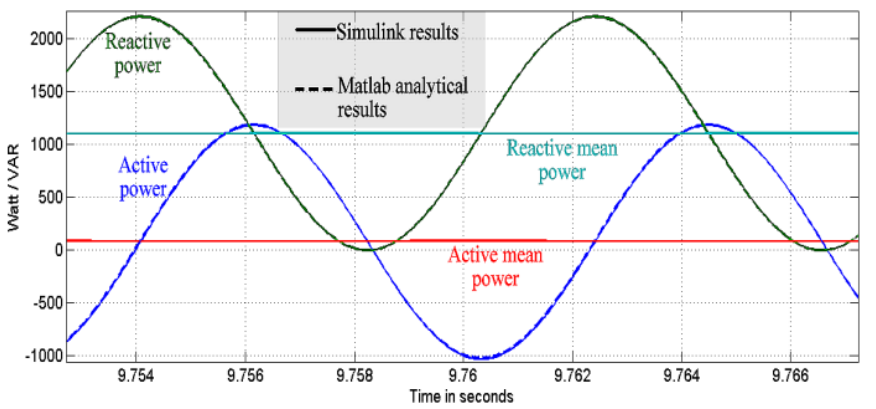

Figure 10. Matlab/Simulink and analytical results

\section{Student assessment}

Steady-state analysis of AC electric circuits is part of the Electric Circuits course given in the first session after two years of general scientific and mathematical fundamentals to undergraduate students at the Department of Applied Sciences of 'Université du Québec en Abitibi-Témiscamingue' in Québec, Canada. The proposed approach was taught to 15 of these students specializing in electromechanical engineering as a reminder of the second chapter in the Electro-technology course, in fall 2010. The students' reactions were generally very positive. They all said that their understanding of the AC power theory was much clearer and much improved. In order to have feedback useful for upgrading the proposed approach, the students were asked to fill out a questionnaire about the theoretical course and the simulation session. The questionnaire consisted of the twelve questions (six positive and six negative) listed in Table 2. The possible answers are given in Table 3 while the results are given in Table 4 . The survey results are summarized in Fig.17. The answers to Question 1 indicate that all students had been taught the instantaneous powers in AC circuits before but 12 out of the 15 claimed that what they learned was not clear. It's also clear the students (14 students) make difference between the proposed and classical approaches.

\section{Table 2. Questionnaire to students}

1. I had been taught instantaneous powers before in Electric Circuits course

2. I had a clear knowledge on the topic before the Electro-technique course

3. The AC power concepts are now clear in my mind

4. I found the approach more comprehensible

5. Numerical simulations help for consolidating my knowledge on the topic

6. The AC circuit analysis in Electric Circuits course needs improvements

7. The approach doesn't bring anything new on the topic

8. I found the approach difficult to understand

9. The classical approach is more suitable than the proposed one

10. I still don't have clear definition of the reactive power

11. I found the approach comprehensible because I knew the topic before

12. There is no difference between classical and new approach

Table 3. List of possible answers to questionnaire

\begin{tabular}{l|l|l}
\hline \hline a. Strongly agree & b. Agree & c. Not sure \\
\hline d. Disagree & e. Strongly disagree & \\
\hline \hline
\end{tabular}

Table 4. Results of the questionnaire answered by students

\begin{tabular}{|c|c|c|c|c|c|c|c|c|c|c|c|c|c|}
\hline \hline \multicolumn{2}{|c|}{} & \multicolumn{10}{c|}{ Questions } \\
\cline { 3 - 16 } & 1 & 2 & 3 & 4 & 5 & 6 & 7 & 8 & 9 & 10 & 11 & 12 \\
\hline \multirow{4}{*}{ Answers } & $\mathrm{a}$ & 15 & 0 & 12 & 13 & 13 & 14 & 0 & 0 & 0 & 0 & 0 & 0 \\
\cline { 2 - 15 } & $\mathrm{b}$ & 0 & 0 & 3 & 2 & 2 & 1 & 0 & 1 & 1 & 1 & 2 & 1 \\
\cline { 2 - 16 } & $\mathrm{c}$ & 0 & 0 & 0 & 0 & 0 & 0 & 0 & 3 & 2 & 2 & 3 & 0 \\
\cline { 2 - 15 } & $\mathrm{d}$ & 0 & 3 & 0 & 0 & 0 & 0 & 0 & 8 & 5 & 5 & 5 & 3 \\
\cline { 2 - 14 } & $\mathrm{e}$ & 0 & 12 & 0 & 0 & 0 & 0 & 15 & 3 & 7 & 7 & 5 & 11 \\
\hline \hline
\end{tabular}

All students were generally satisfied (13 strongly agreed and 2 agreed) with the novel approach and the related numerical simulations provided with Matlab/Simulink tool. Another interesting finding was that all students suggested that changes should be made to the existing Electrical engineering courses. These changes would eliminate some of the redundancies of the same topic in the two different courses (Electric circuits and Electro-technology courses for example). The Frequency and cumulative percentage of students' answers are illustrated in Table 5 and Figure 11.

\section{Conclusion}

The paper proposes a new approach and methodology for improving the definition and teaching of instantaneous powers in steady-state single-phase alternating-current (AC) networks. Its reliability is illustrated by the effectiveness of the results obtained for a few analytical simulation examples with elementary AC loads and by the positive answers and reactions to a questionnaire used as a student assessment. Finally, a physical prototype of instantaneous power metering system is now being built in order to enhance student intuition and help students gain a better understanding and consolidate their knowledge of power analysis of AC circuits.

\section{REFERENCES}

[1] R. C. Dorf, J. A. Svoboda, Electric Circuits, $7^{\text {th }}$ Edition, John Wiley and sons, 2006

[2] James W. Nilson, Susan A. Riedel, Electric Circuits, $6^{\text {th }}$ Edition, Prentice Hall, 2001

[3] J. David Irwin, R. Mark Nelms, Basic Engineering Circuits Analysis, John Wiley and sons, 2005

[4] J.L. Kirtley Jr., AC Power Flow in Linear Networks, Introduction to Power Systems, Class Notes, Chapter 2, Massachusetts Institute of Technology, USA, March 12, 2003

[5] C. P. Steinmetz, Theory and Calculation of Alternating Current Phenomena, McGraw Publishing Company, New York, 1908

[6] M.A Slonim, J.D. Van Wyk, "Power components in a system with sinusoidal and non-sinusoidal voltages and/or currents," IEE Proceedings, vol. 135, no. 2, March 1988, pp. 76-84

[7] T. Neffati, Électricité Générale, Analyse et Synthèse des Circuits, $2^{\circ}$ édition Dunod, Paris, 2008

[8] A. Nabae, and T. Tanaka, "A new Definition of Instantaneous ActiveReactive Current and Power Based on Instantaneous Space Vectors on Polar Coordinates in Three-Phase Circuits,', IEEE Transactions on Power Delivery, vol.11, no.3, July 1996, pp.1238-1243

[9] P.Filipski "A New approach to reactive current and reactive power measurement in non-sinusoidal systems," IEEE Transactions on Instrumentation and Measurement, 1980, IM-29, no.4, pp. 423-426

[10]S. Fryze, "Active, Reactive and Apparent Powers in Network with Nonsinusoidal Waveforms of Voltage and current", ETZ, vol.53, no.25, pp. 596-99, no.26, pp. 625-27, no. 27, pp.700-702, 1932 (in German)

[12]H. Akagi, Y.Kanazawa, and A. Nabae, "Instantaneous reactive power compensators comprising switching devices without energy storage components,' IEEE Transactions on Industry Applications, vol.IA-20, no.3, May/June 1984 , pp. 625-630

[13] T. Furuhashi, S. Okuma, and Y. Uchikawa, "A study on the theory of instantaneous reactive power," IEEE Transactions on Industrial Electronics, vol. 37, no.1, February 1990, pp. 86-90 